\title{
Gravitino thermal production revisited and a new cosmological scenario of gauge mediation
}

\author{
Hiraku Fukushima ${ }^{a, b}$ and Ryuichiro Kitano ${ }^{a, c}$ \\ ${ }^{a}$ KEK Theory Center, \\ Tsukuba 305-0801, Japan \\ ${ }^{b}$ Department of Physics, Tohoku University, \\ Sendai 980-8578, Japan \\ ${ }^{c}$ Department of Particle and Nuclear Physics, \\ The Graduate University for Advanced Studies (Sokendai), \\ Tsukuba 305-0801, Japan \\ E-mail: fukushima@tuhep.phys.tohoku.ac.jp, Ryuichiro.Kitano@kek.jp
}

\begin{abstract}
We present a new scenario of gravitino dark matter which is compatible with the thermal leptogenesis. We confirm by an explicit calculation in supergravity that the relic abundance of thermally produced gravitino becomes insensitive to the reheating temperature once the temperature of the Universe exceeds the mass scale of the messenger fields. In such a situation, the correct baryon to dark matter ratio can be obtained by thermal leptogenesis when the reheating temperature after inflation is high enough. We demonstrate in a concrete model of gauge mediation that the correct abundance of gravitino and baryon asymmetry can be reproduced by considering the late-time entropy production from the decay of the SUSY-breaking pseudo-moduli field. The scenario is realized when the gravitino mass is $100 \mathrm{MeV} \lesssim m_{3 / 2} \lesssim 1 \mathrm{GeV}$, and the messenger mass scale is $10^{6} \mathrm{GeV} \lesssim M_{\text {mess }} \lesssim 10^{9} \mathrm{GeV}$.
\end{abstract}

Keywords: Supersymmetry Phenomenology

ArXiv EPRINT: 1311.6228 


\section{Contents}

1 Introduction $\quad 1$

2 Gravitino thermal production in GMSB revisited 3

2.1 Gravitino thermal production in GMSB 3

2.2 The gravitino relic abundance 8

3 A new scenario of gravitino dark matter $\quad 9$

$\begin{array}{ll}3.1 \text { Compatibility with thermal leptogenesis } & 10\end{array}$

$\begin{array}{lll}3.2 & \text { Late-time entropy release } & 10\end{array}$

$\begin{array}{ll}3.3 & \text { Demonstration in a simple model of gauge mediation } \\ & 11\end{array}$

$\begin{array}{ll}3.4 & \text { Comments on a light higgsino } \\ \end{array}$

$\begin{array}{lll}4 & \text { Summary } & 13\end{array}$

$\begin{array}{ll}\text { A Pseudo-moduli interactions with the MSSM fields } & 15\end{array}$

$\begin{array}{ll}\text { B } \mu \text {-problem and a light higgsino } & 16\end{array}$

\section{Introduction}

The existence of dark matter (DM) is the clearest hint to physics beyond the Standard Model (SM). Among various candidates to explain the unknown component of the Universe, the hypothesis of gravitino dark matter is very attractive as the gravitino always exists in supersymmetric (SUSY) theories and is often the lightest superparticle (LSP) since its mass is suppressed by the Planck scale. The gauge mediated SUSY breaking (GMSB) scenario [1-10] is an explicit realization of the gravitino LSP while the superpartners of the SM particles can be much heavier due to the SM gauge interactions.

In the GMSB models, gravitinos are produced in the early Universe from the thermal bath of the particles in the minimal supersymmetric standard model (MSSM). The production process is more effective at high temperatures, and thus the relic abundance is proportional to the reheating temperature after inflation [12-21], $\Omega_{\mathrm{DM}} \propto T_{R}$. This gives an upper bound on $T_{R}$ so as not for the gravitino abundance to exceed the observed DM abundance, $\Omega_{\mathrm{DM}} h^{2} \simeq 0.1$. The upper bound is $T_{R} \lesssim 10^{6} \mathrm{GeV}$ for $m_{3 / 2} \sim 1 \mathrm{GeV}$ and it becomes more severe for a lighter gravitino. It is, therefore, difficult to realize the gravitino DM compatible with the thermal leptogenesis [22], where the maximal baryon asymmetry is also proportional to $T_{R}$. In order to explain the baron asymmetry of the Universe, we need $T_{R} \gtrsim 10^{9} \mathrm{GeV}[23-26] .{ }^{1}$ The ratio $\Omega_{\mathrm{DM}} / \Omega_{B}$ is predicted to be too large compared to

\footnotetext{
${ }^{1}$ See ref. [51] for a recent study on the gravitino LSP scenario with a high reheating temperature taking into account the results from the LHC experiments.
} 
the observed one, i.e., $\Omega_{\mathrm{DM}} / \Omega_{B} \gg 5$. The late-time entropy production do not help this situation since both the baryon and DM are diluted while fixing the ratio, $\Omega_{\mathrm{DM}} / \Omega_{B}$.

The production rate of gravitino has been calculated in the literatures by using the supergravity Lagrangian, which should be correct at low energy. However, it has been argued in ref. [28], those estimates should be modified in GMSB models for a temperature higher than the messenger scale $M_{\text {mess }}$. The authors of ref. [28] evaluated the gravitino production rate using the Lagrangian of global SUSY, and found that for temperature $T \gg$ $M_{\text {mess }}$, the rate is suppressed by $\sim M_{\text {mess }}^{2} / T^{2}$ compared to the supergravity calculation. If this is the case, the gravitino relic abundance becomes insensitive to $T_{R}$ for $T_{R} \gg M_{\text {mess }}$. The calculations in global SUSY should match the supergravity ones for energies higher than the gravitino mass at the leading order in the $1 / M_{\mathrm{pl}}$ expansion.

Although the statement in ref. [28] is clear in terms of global SUSY, it seems somewhat obscure in the supergravity description. In the global SUSY case, the MSSM fields couple to the goldstino (the longitudinal component of the gravitino) only though the loops of the messenger fields. The production rate is, therefore, significantly modified when the energy goes beyond the mass of the messenger fields. On the other hand, in the supergravity Lagrangian, there are contact derivative interactions between the gravitino and the supercurrent made of the MSSM fields, which lead a growth of the amplitude as energy increases. Therefore, in this description, there is no apparent reason for the gravitino production to be suppressed above the messenger scale $[29,30]$.

In this paper, in order to offer a comprehensive view about the gravitino thermal production, we explicitly calculate a gravitino production process both with a global SUSY Lagrangian and a supergravity Lagrangian, independently. We confirm the suppression of the gravitino production rate both in global SUSY and supergravity for $\sqrt{s}>M_{\text {mess }}$ even though there is a contact interaction term in the supergravity Lagrangian. It is found that the loop diagrams involving messenger fields in the supergravity calculation cancel the tree-level amplitude at a high energy region. The result agrees with the intuition from the goldstino equivalence. The results indicate that the relic abundance of the gravitino is proportional to the messenger scale, $\Omega_{\mathrm{DM}} \propto M_{\text {mess }}$ rather than $T_{R}$ for $T_{R} \gg M_{\text {mess }}$. Therefore, in this occasion, there is no reason to abandon thermal leptogenesis. Given that the gravitino abundance does not depend on $T_{R}$, the ratio $\Omega_{\mathrm{DM}} / \Omega_{B}$ can be fixed to the observed value, $\sim 5$, with a suitable $T_{R}$.

Although the observed DM-baryon ratio can be explained by the thermal leptogenesis, the scenario requires a late-time entropy production by some mechanism, because the produced amount of gravitino is still larger than the observation, $\Omega_{\mathrm{DM}} h^{2} \gg 0.1$, in order to explain the $\Omega_{\mathrm{DM}} / \Omega_{B}$ ratio. Interestingly, we already have a source of the entropy production in GMSB models; there is a pseudo-moduli field in generic low-energy SUSY breaking models, which can supply a large amount of entropy by its decay. We demonstrate the scenario in a simple model of gauge mediation and confirm that the scenario indeed works as the mechanism to produce the right amount of the gravitino DM.

The sketch of the scenario is as follows; the reheating of the Universe occurs at a high $T_{R}$ so that the gravitino abundance is independent of $T_{R}$. With an appropriate reheating temperature, the ratio of energy densities $\Omega_{\mathrm{DM}} / \Omega_{B}$ can be fixed at the observed value, 
$\Omega_{\mathrm{DM}} / \Omega_{B} \sim 5$, after the reheating process. Later, the SUSY breaking pseudo-moduli starts coherent oscillation about the minimum of the potential, and the oscillation energy eventually dominates the Universe. A sizable amount of entropy is released by the subsequent decay, and the pre-existing gravitinos and baryons are diluted by a same amount to realize the observed values.

Throughout our analysis, the SUSY scale is assumed to be $M_{\mathrm{SUSY}} \simeq 5 \mathrm{TeV}$ to realize $m_{h}=125 \mathrm{GeV}$ [11] within the MSSM. Although it sounds difficult to confirm the scenario by the LHC experiments, the framework we use predicts a relatively small $\mu$-term and thus there is a light higgsino with $m_{\tilde{h}} \sim \mathcal{O}(100) \mathrm{GeV}$. We explain this point in appendix B. Such a light higgsino may be within the reach of future experiments such as at an International Linear Collider (ILC). Since the life-time of higgsino can be as long as $O(1)$ sec, we check the constraints from the Big-Bang nucleosynthesis (BBN) and find that the light higgsino is cosmologically safe if the gravitino mass is less than $\sim 500 \mathrm{MeV}$.

\section{Gravitino thermal production in GMSB revisited}

The gravitino production rate has been calculated by using the supergravity Lagrangian [12-21], which leads the result that the abundance is proportional to $T_{R}$. In GMSB models, the production is dominated by that of the longitudinal mode which can be evaluated by identifying the longitudinal mode as the goldstino in the global SUSY Lagrangian. Moreover, in GMSB models, one can use a framework of a linearly realized SUSY breaking model with a singlet superfield $S$, whose $F$-component VEV breaks the SUSY.

An explicit calculation of the goldstino production shows that the goldstino relic abundance is not necessarily proportional to $T_{R}[28]$, which contradicts with the estimation in supergravity. We examine this apparent contradiction by calculating the scattering amplitudes of goldstino/gravitino production process both with a global SUSY Lagrangian and a supergravity Lagrangian. We confirm that the supergravity result should be modified at high energy.

\subsection{Gravitino thermal production in GMSB}

Calculation in supergravity Lagrangian. Here we briefly review why the gravitino relic abundance is determined by the reheating temperature $T_{R}$. Gravitinos are produced from the scattering process of the MSSM fields and the amplitudes are calculated by using the supergravity Lagrangian,

$$
\mathcal{L}_{\text {sugra }}^{\mathrm{MSSM}} \ni-\frac{1}{\sqrt{2} M_{\mathrm{pl}}}\left(D_{\nu} \phi_{i}\right)^{*} \bar{\psi}_{3 / 2 \mu} \gamma^{\nu} \gamma^{\mu} P_{L} \psi_{i}-\frac{i}{4 M_{\mathrm{pl}}} \bar{\lambda}^{a} \gamma^{\mu}\left[\gamma^{\nu}, \gamma^{\rho}\right] \psi_{3 / 2 \mu} F_{\nu \rho}^{a}+\text { h.c. },
$$

where the gravitino field is denoted by $\psi_{3 / 2 \mu}$. The gravitino has the tree-level interactions with all the chiral multiplets $\left(\phi_{i}, \psi_{i}\right)$ or gauge multiplets $\left(A_{\mu}^{a}, \lambda^{a}\right)$ in the MSSM and the form of interactions is uniquely fixed by local SUSY.

For the gravitino production, there are ten two-body processes involving left-handed quarks $\left(q_{i}\right)$, squarks $\left(\tilde{q}_{i}\right)$, gauginos $\left(\lambda^{a}\right)$ and the gauge bosons $\left(A^{a}\right)$, which are called processes $\mathrm{A}$ to $\mathrm{J}$ in the literatures $[12-14,16,17]$. In the literatures the QCD processes are 

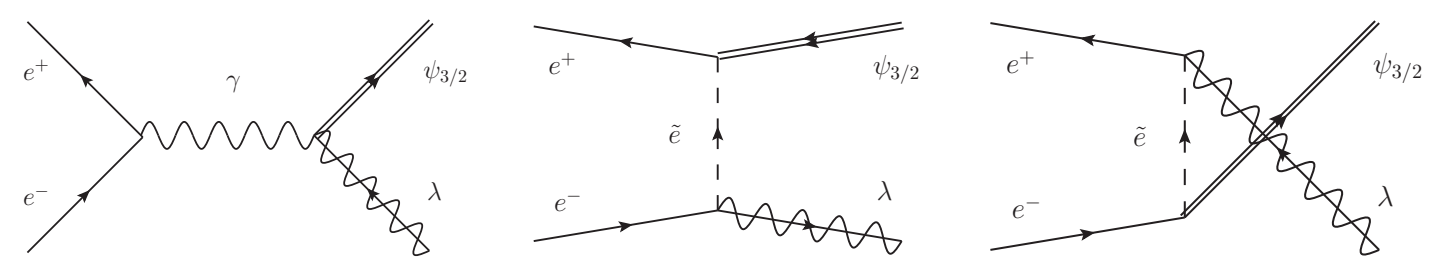

Figure 1. Gravitino production process $e^{-} e^{+} \rightarrow \lambda \psi_{3 / 2}$.

discussed in detail because they are the dominant processes. Here we focus on a particular process $e^{-} e^{+} \rightarrow \lambda \psi_{3 / 2}$ (called process I in the literatures) for simplicity. The tree-level diagrams are shown in figure 1.

The scattering amplitude is calculated by the supergravity Lagrangian in eq. (2.1). Among the polarized amplitudes, the following turns out to have the highest power in the center-of-mass energy, $\sqrt{s}$, and thus dominates at high energies,

$$
\mathcal{M}_{e^{-} e^{+} \rightarrow \lambda \psi_{3 / 2}}^{(\uparrow \downarrow \uparrow)}=\frac{e m_{\lambda}}{\sqrt{6} m_{3 / 2} M_{\mathrm{pl}}} \sqrt{s} \sin \theta
$$

where arrows in the parenthesis represent the spins of the electron, the positron, the gaugino and the gravitino, respectively. The angle $\theta$ is the production angle in the center-of-mass frame. The gauge coupling of QED is denoted by $e$. Although each of $s^{-}, t$ - and $u$-channel diagrams has an energy dependence of $O(s)$, they are canceled out when combined, remaining the energy dependence of $O(\sqrt{s})$. The above contribution is from the longitudinal component of the gravitino whose wave function is approximately proportional to $\sqrt{s} / m_{3 / 2}$ with $m_{3 / 2}$ the gravitino mass.

In order to estimate the relic abundance of the gravitino, we should calculate the reaction rate which is proportional to the square of the amplitude,

$$
\Gamma_{e^{-} e^{+} \rightarrow \lambda \psi_{3 / 2}}(T) \propto \frac{m_{\lambda}^{2}}{m_{3 / 2}^{2} M_{\mathrm{pl}}^{2}} T^{3},
$$

where the temperature dependence is determined by dimensional analysis. The key is the cubic dependence on $T$. If the reaction rate depends on the temperature with a higher power than the Hubble parameter $H(T) \propto T^{2}$, the resultant gravitino abundance is fixed at high temperature, $T_{R}$. In contrast, if the power is lower than $H(T)$, the yield is fixed by the lowest temperature. If the process $e^{-} e^{+} \rightarrow \lambda \psi_{3 / 2}$ is effective and eq. (2.3) is valid for an arbitrary temperature, the gravitino abundance is determined by $T_{R}$.

Goldstino analysis. In GMSB models, effects of SUSY breaking are transmitted to the MSSM sector through the messenger loop diagrams. A superpotential of the following form is usually assumed,

$$
W=\lambda S f \bar{f} .
$$

SUSY is broken by the $F$-component of the singlet superfield $S . f$ and $\bar{f}$ represent the messenger superfields which have SM gauge charges. If $F_{S}$ is the only source of the 

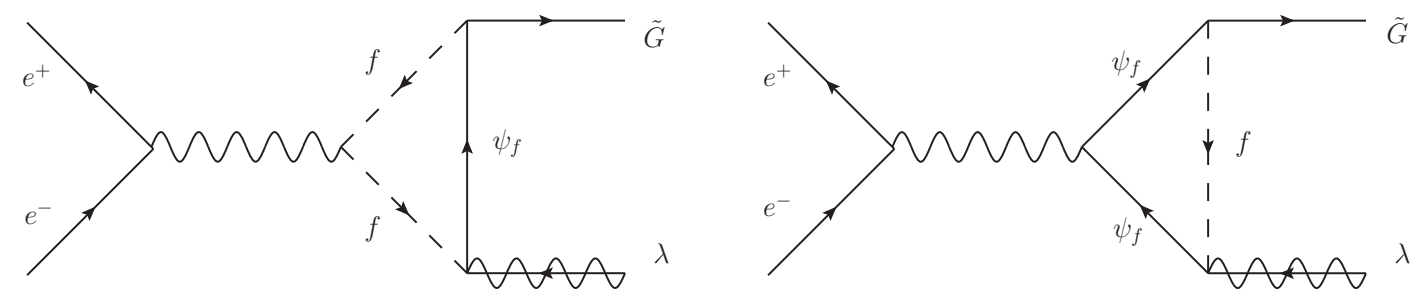

Figure 2. Goldstino production process $e^{-} e^{+} \rightarrow \lambda \tilde{G}$.

SUSY breaking, the fermion component of $S$ (we call it $\psi_{S}$ ) is the goldstino $\tilde{G}$, which is absorbed into the longitudinal component of the gravitino. In general, there are additional sources of SUSY breaking from the $F$-components of other chiral multiplets. In that case, the goldstino is composed of the liner combination of the fermions which belong to the multiplets whose $F$-components develop VEVs,

$$
\tilde{G}=\frac{F_{S}}{F} \psi_{S}+\sum_{i} \frac{F_{i}}{F} \psi_{i}
$$

where $F=\sqrt{\left|F_{S}\right|^{2}+\sum_{i}\left|F_{i}\right|^{2}}$. Therefore, the amplitude for the goldstino production is given by rescaling that for $\psi_{S}$ by a factor $F_{S} / F$. Unlike the gravitino in the supergravity Lagrangian, the goldstino does not couple directly to the MSSM fields. The goldstino is produced through the messenger loop diagrams shown in figure 2 . We expect that the scattering amplitude of the process $e^{-} e^{+} \rightarrow \lambda \tilde{G}$ coincides that of the gravitino production in eq. (2.2).

By explicitly evaluating these diagrams, however, a different result from supergravity estimation comes out. For the same process and the same polarization to eq. (2.2), the scattering amplitude is calculated to be

$$
\begin{aligned}
\mathcal{M}_{e^{-} e^{+} \rightarrow \lambda \tilde{G}}^{(\uparrow \downarrow \uparrow)} & =-\frac{2 \sqrt{2} e^{3} \lambda}{(4 \pi)^{2}} \frac{F_{S}}{F} M_{\text {mess }} C_{0}\left(\sqrt{s}, M_{\text {mess }}\right) \sqrt{s} \sin \theta \\
& =-\frac{2 e m_{\lambda} M_{\text {mess }}^{2}}{\sqrt{6} m_{3 / 2} M_{\mathrm{pl}}} C_{0}\left(\sqrt{s}, M_{\text {mess }}\right) \sqrt{s} \sin \theta
\end{aligned}
$$

where $M_{\text {mess }}=\lambda\langle S\rangle$ is the messenger mass scale. We have translated the parameters of global SUSY, $\lambda$ and $\langle S\rangle$, to the parameters of the supergravity, $m_{3 / 2}$ and $M_{\mathrm{pl}}$ by using the formulae in GMSB:

$$
m_{\lambda}=\frac{2 e^{2}}{(4 \pi)^{2}} \frac{F_{S}}{\langle S\rangle}
$$

and

$$
m_{3 / 2}=\frac{F}{\sqrt{3} M_{\mathrm{pl}}} .
$$

The function $C_{0}\left(\sqrt{s}, M_{\text {mess }}\right)$ is the $C$-function defined in ref. [27],

$$
C_{0}\left(\sqrt{s}, M_{\text {mess }}\right)=\int_{0}^{1} d x \frac{1}{s(1-x)} \log \left[1-\frac{s}{M_{\text {mess }}^{2}} x(1-x)-i \epsilon\right] .
$$


In a low energy limit, $\sqrt{s} \ll M_{\text {mess }}, C_{0}$ is approximately given by $C_{0} \simeq-1 / 2 M_{\text {mess }}^{2}$ and reproduces the result of supergravity calculation in eq. (2.2). However, for $\sqrt{s} \gg M_{\text {mess }}$, $C_{0}$ scales as $1 / s$ up to a logarithmic factor.

If the external energies are lower than the messenger mass scale, i.e., for $T<M_{\text {mess }}$, the reaction rate depends on the temperature as $\propto T^{3}$,

$$
\Gamma_{e^{-} e^{+} \rightarrow \lambda \tilde{G}}(T) \propto \frac{m_{\lambda}^{2}}{m_{3 / 2}^{2} M_{\mathrm{pl}}^{2}} T^{3}, \quad \text { for } T \ll M_{\text {mess }},
$$

which reproduces the result of the supergravity calculation in eq. (2.3). Here we again squared the amplitude and fixed the temperature dependence by dimensional analysis. However, for $T>M_{\text {mess }}$, the reaction rate is suppressed by $\sim M_{\text {mess }}^{2} / T^{2}$ compared to eq. (2.11), namely

$$
\Gamma_{e^{-} e^{+} \rightarrow \lambda \tilde{G}}(T) \propto \frac{m_{\lambda}^{2} M_{\mathrm{mess}}^{2}}{m_{3 / 2}^{2} M_{\mathrm{pl}}^{2}} T, \quad \text { for } T \gg M_{\mathrm{mess}} .
$$

The point is that the temperature dependence of $\Gamma_{e^{-} e^{+} \rightarrow \lambda \tilde{G}}(T) / H(T)$ gets suppressed as $1 / T$ at high temperatures, which makes the goldstino relic abundance irrelevant to the reheating temperature. Rather, the abundance is determined by the messenger mass scale.

Supergravity calculation in GMSB. We observe a difference between the two amplitudes, eq. (2.2) and eq. (2.7). One of them should be modified at high energy, $\sqrt{s} \gg M_{\text {mess }}$, if we believe in the goldstino equivalence.

We find that the modification appears in the supergravity calculation. In GMSB models, there are messenger fields, which potentially affect the gravitino production process. In fact, they contribute to the gravitino production process $e^{-} e^{+} \rightarrow \lambda \psi_{3 / 2}$ through the one-loop diagrams shown in figure 3. Even though they are diagrams at the one-loop level, they cannot be neglected compared to the tree-level ones in figure 1 since the gaugino mass in eq. (2.2) is at the one-loop order in GMSB models. Note here that the diagrams in figure 3 are not the microscopic description of the first diagram in figure 1. Both diagrams exist as independent ones in supergravity. The explicit calculation shows

$$
\mathcal{M}_{e^{-} e^{+} \rightarrow \lambda \psi_{3 / 2}}^{(\uparrow \downarrow \uparrow \uparrow)}(\text { one loop })=-\frac{e m_{\lambda}}{\sqrt{6} m_{3 / 2} M_{\mathrm{pl}}} \sqrt{s} \sin \theta\left[2 M_{\text {mess }}^{2} C_{0}\left(\sqrt{s}, M_{\text {mess }}\right)+1\right]
$$

where $C_{0}$ is again the $C$-function in eq. (2.10). The dots in figure 3 represent insertions of $F_{S}$, and we used eq. (2.8) to derive the above formula. A few comments are in order. At a lower energy than the messenger mass scale, the messenger fields can be integrated out and absent in the low energy theory. The gravitino interactions are then completely read off from the supergravity Lagrangian of the MSSM fields (2.1). The supergravity prediction in eq. (2.2), therefore, should not be altered for $\sqrt{s} \ll M_{\text {mess }}$. The additional contribution (2.13) indeed respects this consideration. The factor, $2 M_{\text {mess }}^{2} C_{0}+1$, in eq. (2.13) goes to zero as $\sqrt{s} \rightarrow 0$, and thus the amplitude is accurately represented by eq. (2.2) at low energy. However, the one-loop contribution becomes comparable to that of tree-level for $\sqrt{s} \gg M_{\text {mess }}$ since the factor, $2 M_{\text {mess }}^{2} C_{0}+1$, approaches to 1 . 


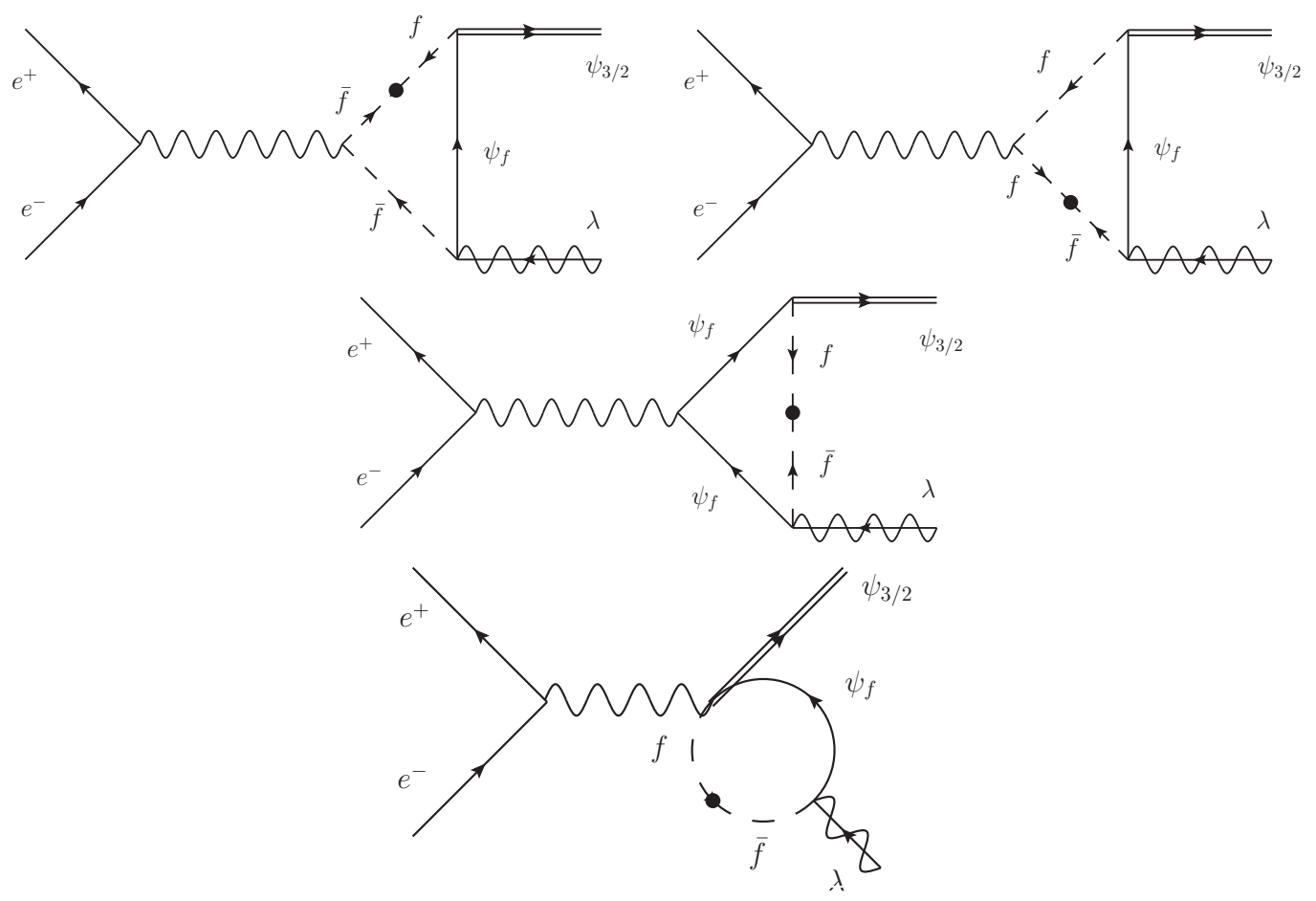

Figure 3. One-loop diagrams for the gravitino production $e^{-} e^{+} \rightarrow \lambda \psi_{3 / 2}$.

Combined with the tree-level contribution (2.2), we confirmed that the growing amplitude at $\sqrt{s} \gg M_{\text {mess }}$ in supergravity is completely cancelled by the one-loop diagrams, and the total supergravity calculation coincides with the result from global SUSY,

$$
\begin{aligned}
\mathcal{M}_{e^{-} e^{+} \rightarrow \lambda \psi_{3 / 2}}^{(\uparrow \downarrow \uparrow \uparrow)} & =\mathcal{M}_{e^{-} e^{+} \rightarrow \lambda \psi_{3 / 2}}^{(\uparrow \downarrow \uparrow)}(\text { tree })+\mathcal{M}_{e^{-} e^{+} \rightarrow \lambda \psi_{3 / 2}}^{(\uparrow \downarrow \uparrow \uparrow)}(\text { one loop) } \\
& =\frac{e m_{\lambda}}{\sqrt{6} m_{3 / 2} M_{\mathrm{pl}}} \sqrt{s} \sin \theta-\frac{e m_{\lambda}}{\sqrt{6} m_{3 / 2} M_{\mathrm{pl}}} \sqrt{s} \sin \theta\left[2 M_{\text {mess }}^{2} C_{0}\left(\sqrt{s}, M_{\text {mess }}\right)+1\right] \\
& =-\frac{2 e m_{\lambda} M_{\text {mess }}^{2}}{\sqrt{6} m_{3 / 2} M_{\mathrm{pl}}} C_{0}\left(\sqrt{s}, M_{\text {mess }}\right) \sqrt{s} \sin \theta .
\end{aligned}
$$

Additional contribution from the tree-level messenger scatterings. For $T>M_{\text {mess }}$, in addition to the scattering processes of the MSSM particles, the goldstino is also produced by scattering processes where the messenger fields are in the external lines. The reaction rate is calculated to be [28]

$$
\begin{aligned}
\Gamma_{\text {messengers } \rightarrow \lambda \tilde{G}}(T) & \propto \lambda^{2}\left(\frac{F_{S}}{F}\right)^{2} T \\
& \propto\left(\frac{4 \pi}{\alpha}\right)^{2} \frac{m_{\lambda}^{2} M_{\text {mess }}^{2}}{m_{3 / 2}^{2} M_{\mathrm{pl}}^{2}} T .
\end{aligned}
$$

As we see from eq. (2.12) and eq. (2.15), the reaction rate of the messenger particles is larger than that of the MSSM particles by a loop-factor since the messenger fields directly couple to the goldstino through the superpotential interaction. 


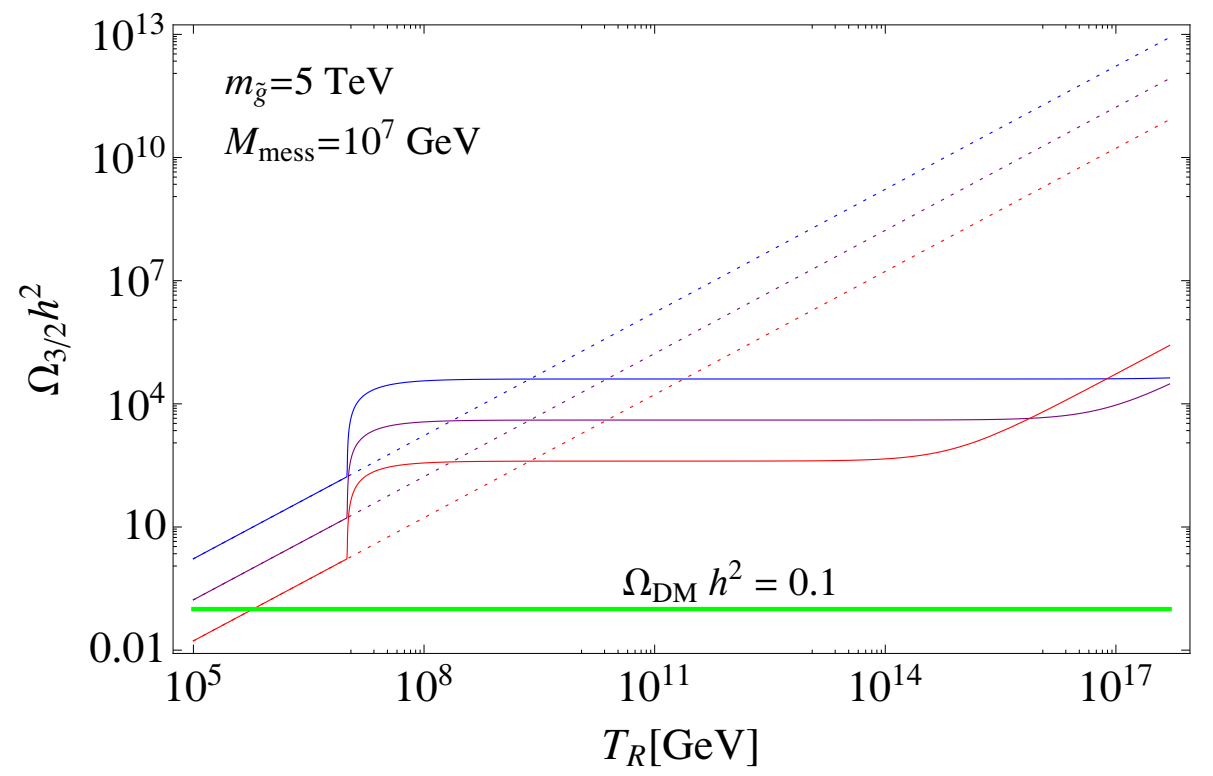

Figure 4. Gravitino relic abundance. Blue, purple, and red lines represent $m_{3 / 2}=100 \mathrm{MeV}$, $m_{3 / 2}=1 \mathrm{GeV}$ and $m_{3 / 2}=10 \mathrm{GeV}$, respectively. The gravitino abundance become insensitive to the reheating temperature for $M_{\text {mess }}<T_{R}$ (solid lines). Dotted lines are naive extrapolations of eq. (2.16). For a very high reheating temperature $\left(T_{R} \gtrsim 10^{14} \mathrm{GeV}\right)$, the transverse mode of the gravitino becomes important.

\subsection{The gravitino relic abundance}

Summarizing the previous subsection, in GMSB models, the gravitino is produced from the scattering processes of the MSSM fields and the messenger fields. Depending on the value of $T_{R}$, the resultant gravitino relic abundance is determined by different values; if $T_{R}<M_{\text {mess }}$, the abundance is fixed by $T_{R}$, and if $T_{R}>M_{\text {mess }}$, it is the messenger mass scale to fix the abundance,

$$
\begin{array}{ll}
\Omega_{3 / 2} h^{2} \simeq 0.45\left(\frac{T_{R}}{10^{6} \mathrm{GeV}}\right)\left(\frac{\mathrm{GeV}}{m_{3 / 2}}\right)\left(\frac{m_{\tilde{g}}}{5 \mathrm{TeV}}\right)^{2} & \left(T_{R}<M_{\text {mess }}\right), \\
\Omega_{3 / 2} h^{2} \simeq 3.7 \times 10^{2}\left(\frac{M_{\text {mess }}}{10^{6} \mathrm{GeV}}\right)\left(\frac{\mathrm{GeV}}{m_{3 / 2}}\right)\left(\frac{m_{\tilde{g}}}{5 \mathrm{TeV}}\right)^{2} & \left(T_{R}>M_{\text {mess }}\right) .
\end{array}
$$

The abundance in eq. (2.17) is not a straightforward replacement of $T_{R}$ to $M_{\text {mess }}$ in eq. (2.16) since the production through the messenger fields are not suppressed by a loop factor.

The estimates so far do not include a contribution of the transverse mode of the gravitino. For a very high reheating temperature, the transverse mode becomes relevant,

$$
\Omega_{3 / 2} h^{2}(\text { transverse }) \simeq 0.53\left(\frac{T_{R}}{10^{13} \mathrm{GeV}}\right)\left(\frac{m_{3 / 2}}{\mathrm{GeV}}\right) .
$$

Including both the longitudinal and the transverse modes, we show the gravitino relic abundance in GMSB with the messenger scale fixed to be $M_{\text {mess }}=10^{7} \mathrm{GeV}$ in figure 4 . 


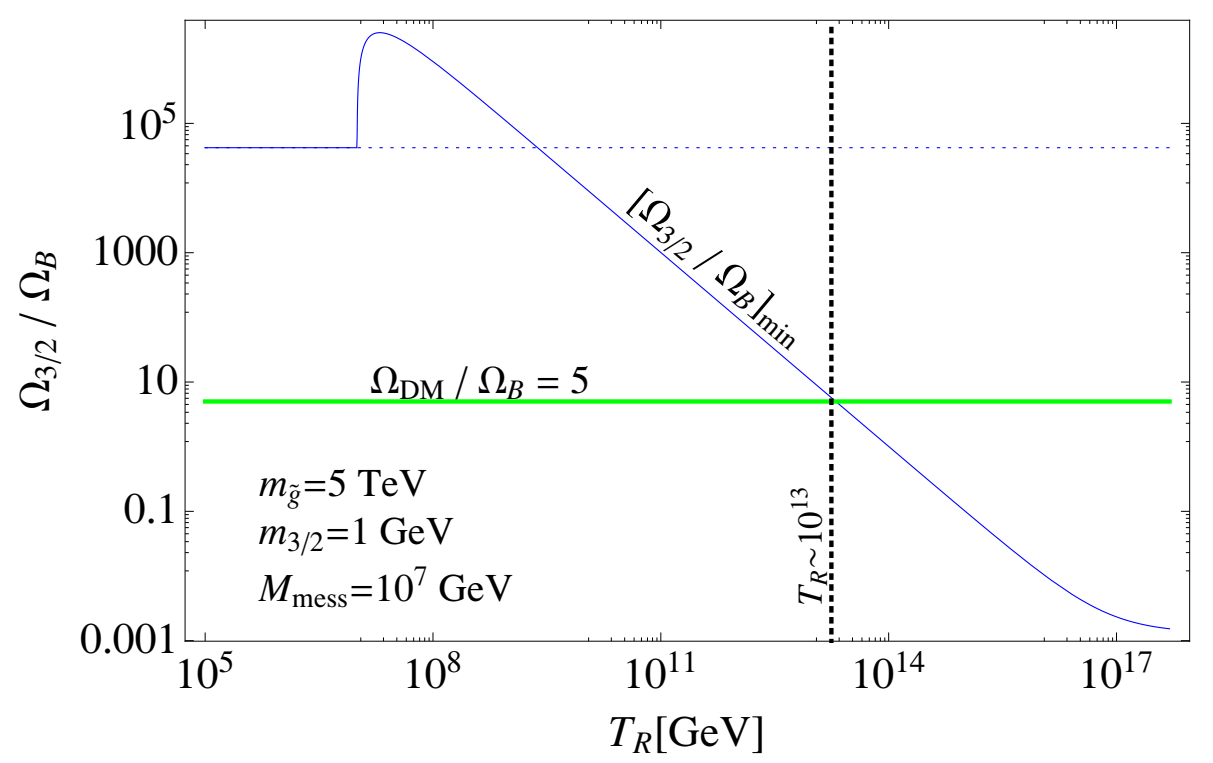

Figure 5. Since the gravitino abundance becomes constant for $M_{\text {mess }}<T_{R}$ whereas the maximum value of $\Omega_{B}$ is always proportional to $T_{R}$, the ratio $\Omega_{3 / 2} / \Omega_{B}$ eventually reaches the observed value as $T_{R}$ becomes higher. We plotted a minimum value of the prediction for $\Omega_{3 / 2} / \Omega_{B}$ as a function of $T_{R}$. We see that the observed value of $\Omega_{\mathrm{DM}} / \Omega_{B}$ can be reproduced for $T_{R} \gtrsim 10^{13} \mathrm{GeV}$.

As we see from the figure, the gravitino relic abundance is predicted to be constant in a wide range of the reheating temperature, but the amount is too large compared to the observed dark matter energy density $\Omega_{\mathrm{DM}} h^{2} \simeq 0.1$. The overproduced gravitinos must be diluted by some mechanism. Although the prediction to $\Omega_{3 / 2}$ is too large, the insensitivity to $T_{R}$ brings us a new scenario of gravitino DM.

\section{A new scenario of gravitino dark matter}

As we have confirmed in the previous section, the gravitino relic abundance becomes insensitive to $T_{R}$ once the temperature of the Universe exceeds the messenger mass scale. The results have a crucial impact on the possible mechanism of baryogenesis. In this section, we present a new cosmological scenario of gauge mediation, where gravitino dark matter and thermal leptogenesis are compatible. The scenario requires a late-time entropy release by some mechanism, which is automatically supplied by the decay of the SUSY breaking pseudo-moduli field. We demonstrate the scenario with a simple model of gauge mediation as an example and see that the scenario actually works.

Throughout the analysis, we assume the SUSY scale to be $M_{\mathrm{SUSY}} \gtrsim 5 \mathrm{TeV}$ and in particular fix the gluino mass to be $m_{\tilde{g}}=5 \mathrm{TeV}$ to account for the Higgs boson mass of $125 \mathrm{GeV}$ within the MSSM in GMSB [11]. Although most of the SUSY particles are then too heavy to be detected at the LHC experiments, the model predicts higgsino to be as light as $m_{\tilde{h}} \sim \mathcal{O}(100) \mathrm{GeV}$. We briefly mention the cosmological constraint on the light higgsino in the last subsection. 


\subsection{Compatibility with thermal leptogenesis}

In a light gravitino scenario, thermal leptogenesis and gravitino DM are thought to be incompatible with each other. The possible maximum amount of baryon asymmetry produced by the thermal leptogenesis is proportional to the reheating temperature [23-26],

$$
\Omega_{B} \lesssim 0.04\left(\frac{T_{R}}{10^{9} \mathrm{GeV}}\right),
$$

which puts a lower bound on $T_{R}\left(T_{R} \gtrsim 10^{9} \mathrm{GeV}\right)$ to realize the observed value $\Omega_{B} \simeq 0.045$. If the gravitino relic abundance is represented as eq. (2.16) for any $T_{R}$, the thermal production of gravitino DM and the thermal leptogenesis are incompatible; even if we assume a late-time entropy production to dilute overproduced gravitino to match the abundance to the observation, baryons are also diluted at the same time and the abundance never reproduces the observation. In other words, the ratio $\Omega_{3 / 2} / \Omega_{B}$ is constant as long as the abundances are both proportional to $T_{R}$, and always larger than the observed ratio, $\Omega_{\mathrm{DM}} / \Omega_{B} \sim 5$.

However, in GMSB, if the reheating temperature is higher than the messenger mass scale, the gravitino relic abundance becomes insensitive to $T_{R}$. Then, the observed ratio of the energy densities, $\Omega_{\mathrm{DM}} / \Omega_{B} \sim 5$, can be realized with thermally produced gravitino and the thermal leptogenesis. We plot the prediction for $\Omega_{3 / 2} / \Omega_{B}$ to visualize the situation in figure 5. If the gravitino abundance is proportional to $T_{R}$ for any $T_{R}$, the theoretical prediction never reaches the observed value $\Omega_{\mathrm{DM}} / \Omega_{B} \sim 5$ (dotted line). However, if the reheating temperature is higher than the messenger scale, $\Omega_{3 / 2}$ becomes independent of $T_{R}$ in GMSB, which allows $\Omega_{3 / 2} / \Omega_{B}$ to achieve the observed value.

\subsection{Late-time entropy release}

The ratio of the energy densities $\Omega_{\mathrm{DM}} / \Omega_{B} \sim 5$ can be realized by thermally produced gravitino and thermal leptogenesis with an appropriate reheating temperature as we saw above. However, as is obvious from figure 4, the predicted gravitino abundance is too large compared to the observation, $\Omega_{\mathrm{DM}} h^{2} \simeq 0.1$. The overproduced gravitino should be diluted by a late-time entropy release by some mechanism. The required amount of dilution is

$$
\begin{aligned}
\Delta_{3 / 2} & \equiv \frac{\Omega_{3 / 2} h^{2}}{\Omega_{\mathrm{DM}} h^{2}} \\
& \simeq 7.5 \times 10^{4}\left(\frac{M_{\text {mess }}}{10^{7} \mathrm{GeV}}\right)\left(\frac{500 \mathrm{MeV}}{m_{3 / 2}}\right)\left(\frac{m_{\tilde{g}}}{5 \mathrm{TeV}}\right)^{2},
\end{aligned}
$$

where $T_{R}>M_{\text {mess }}$ is assumed.

Actually, a source of entropy production is already incorporated in the scenario: the scalar component of the singlet superfield $S$, which is called the pseudo-moduli field. In the early Universe, it is possible that the pseudo-moduli is displaced from the vacuum and starts oscillation around the minimum. Since the pseudo-moduli is massless at tree-level and gets mass only through the quantum effects, it is often much lighter than the SUSY breaking scale, $\sqrt{F}$, and is long-lived if there is a weakly coupled description for the SUSY breaking sector. In such a case, the pseudo-moduli can eventually dominate the energy density of the Universe, and a sizable amount of entropy is produced from its decay. 


\subsection{Demonstration in a simple model of gauge mediation}

The model. We study a low-energy effective theory of O'Raifeartaigh type SUSY breaking model coupled with the messenger fields:

$$
\begin{aligned}
K & =f^{\dagger} f+\bar{f}^{\dagger} \bar{f}+S^{\dagger} S-\frac{\left(S^{\dagger} S\right)^{2}}{\Lambda^{2}}+\cdots, \\
W & =m^{2} S-\lambda S f \bar{f}+c
\end{aligned}
$$

where $S$ is a gauge singlet superfield called the SUSY breaking pseudo-moduli. The messenger superfields are demoted by $f$ and $\bar{f}$. There is an $R$-symmetry where the charge assignment is $R(S)=2$ and $R(f \bar{f})=0$. If the $R$-symmetry is unbroken, $S$ is stabilized at $S=0$ where we cannot integrate out the messenger fields. Once we turn on the supergravity effects, however, the $R$-symmetry is explicitly broken by the supergravity correction represented by the constant term $c$, which destabilizes the origin and creates the SUSY breaking vacuum at $\langle S\rangle \sim \Lambda^{2} / M_{\mathrm{pl}}[31]$, where $M_{\mathrm{pl}} \simeq 2.4 \times 10^{18} \mathrm{GeV}$ is the reduced Planck scale.

Since there is also a SUSY preserving minimum at $S=0$ where the messenger fields condense, the SUSY breaking vacuum is a meta-stable state. For a realistic cosmology, the $S$ field should stay away from the SUSY vacuum in the course of cosmological evolution.

Cosmological evolution of $\boldsymbol{S}$. Cosmological evolution of the pseudo-moduli in the model in eqs. (3.4) and (3.5) is examined in detail in refs. [33-37]. It has been found that the SUSY breaking minimum is preferred to the SUSY preserving one for a wide region of the parameter space even if the messenger particles enter the thermal equilibrium. ${ }^{2}$ Also, with an appropriate initial condition the pseudo-moduli start oscillation around the SUSY breaking vacuum and the oscillation energy dominates the energy density of the Universe.

We define the dilution factor $\Delta$ due to the entropy release from the decay of the pseudo-moduli as

$$
\frac{1}{\Delta} \equiv \frac{s_{\text {inf }}}{s_{S}+s_{\text {inf }}} \simeq \operatorname{Min}\left[1, \frac{s_{\text {inf }}}{s_{S}}\right]
$$

where $s_{\text {inf }}$ and $s_{S}$ represent the entropy densities produced by the decays of the inflaton and $S$, respectively. If $\Delta>1, \Delta$ is well approximated by

$$
\Delta \simeq \frac{s_{S}}{s_{\text {inf }}}=\frac{4}{3 T_{d}} \cdot \frac{\rho_{S}}{s_{\text {inf }}},
$$

where $\rho_{S}$ is the energy density of $S$ and $T_{d}$ is a decay temperature of the pseudo-moduli, which is defined by

$$
T_{d} \equiv\left(\frac{\pi^{2} g_{*}}{90}\right)^{-1 / 4} \sqrt{M_{\mathrm{pl}} \Gamma_{S}} .
$$

The total decay width of $S$ is denoted as $\Gamma_{S}$. The formulae of $\Gamma_{S}$ and $T_{d}$ are found in appendix A.

\footnotetext{
${ }^{2}$ The vacuum selection is discussed in the literatures [38-47]. The present model had been thought to be problematic because the $S$ field tends to fall into the SUSY preserving vacuum by a finite temperature potential.
} 
If the magnitude of dilution factor $\Delta$ coincides $\Delta_{3 / 2}$ in eq. (3.3), the overproduced gravitinos are diluted to realize the observed dark matter abundance, $\Omega_{\mathrm{DM}} h^{2} \simeq 0.1$. In order to realize the right amount of baryons, $\Omega_{B} \simeq 0.045$, at the same time, we need an appropriate reheating temperature. Since the baryon asymmetry is also diluted by the entropy production, the reheating temperature should be high enough to produce abundant baryons in advance, namely $10^{9} \times \Delta_{3 / 2} \lesssim T_{R}$ is required in the scenario. We show the required set of the dilution factor $\left(\Delta_{3 / 2}\right)$ and the reheating temperature $\left(T_{R}\right)$ in $m_{3 / 2}$ vs $M_{\text {mess }}$ plane in figure 6 .

In the present set-up, there exists a parameter region where the dark matter and the baryon asymmetry are explained by thermally produced gravitino and thermal leptogenesis simultaneously (blue and green regions), with an appropriate combination of $\Delta$ and $T_{R}$.

In order to estimate the magnitude of the dilution factor from the decay, we numerically solved the equation of motion of the pseudo-moduli with the initial condition set at the inflaton dominated era. The results depend on the initial location of the $S$ field which can be far away from the origin depending on the inflation model and the coupling between $S$ and the inflaton [33,34]. In this study, we choose the initial position of $S$ to be $\Lambda$ or $M_{\mathrm{pl}}$ for illustration. The results are shown in figure 7 . As we see from the figure, by choosing an appropriate value of the initial condition of $S$ from between $\Lambda$ and $M_{\mathrm{pl}}$, the required amount of entropy can be supplied from the oscillation energy everywhere in the blue and green regions in figure 6 ; we have confirmed that required entropy production can be obtained in this model.

Non-thermal gravitino production. While the dark matter is explained by thermally produced gravitino in the blue and green regions in figure 6, gravitinos are also produced non-thermally by the rare decay $S \rightarrow \psi_{3 / 2} \psi_{3 / 2}$. We calculate the non-thermally produced gravitino abundance in appendix $\mathrm{A}$ and found that the abundance coincides the observed dark matter abundance with $m_{3 / 2} \sim 2 \mathrm{GeV}$. Taking into account possible theoretical errors, we show the parameter region where $0.03 \lesssim \Omega_{3 / 2}^{\mathrm{NT}} h^{2} \lesssim 0.3$ is predicted as a green band in figure 6 .

\subsection{Comments on a light higgsino}

So far we have studied a new cosmological scenario with a high SUSY scale $M_{\text {SUSY }} \gtrsim 5 \mathrm{TeV}$ in order to realize a $125 \mathrm{GeV}$ Higgs boson mass. If all the SUSY particles are as heavy as $5 \mathrm{TeV}$, it is difficult to confirm the scenario by the LHC experiments. However, it is possible that the $\mu$-parameter in the MSSM is much smaller than other superparticle masses. In the GMSB model we used for the cosmological study there is a natural solution to the $\mu$-problem (we mention the prescription in appendix B). The model predicts a light higgsino with its mass of $\mathcal{O}(100) \mathrm{GeV}$. The $\mu$-term is generated by a direct coupling between SUSY breaking chiral multiplet and Higgs multiplets assumed at the cutoff scale $\Lambda$, which results in a relatively small $\mu$-term compared to Higgs soft mass parameters. For a cosmologically favorable region of the gravitino mass, the lightest higgsino does not decay inside the detector. In that case, searches for mono-jet processes at LHC or mono-photon ones at the ILC will be able to find the light higgsino. 


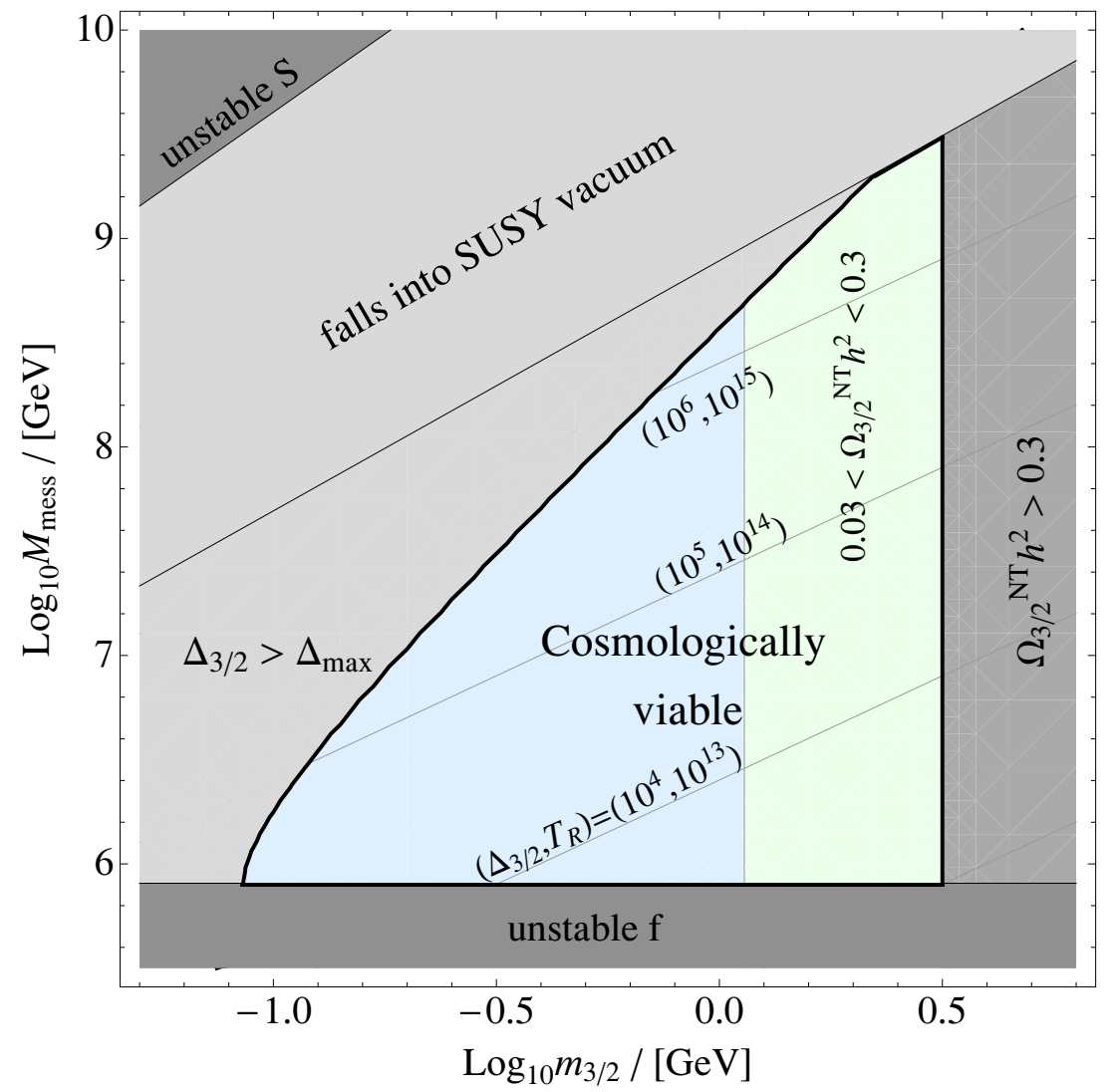

Figure 6. Required amount of the dilution factor $\left(\Delta_{3 / 2}\right)$ and the reheating temperature $\left(T_{R}\right)$ to realize the observation $\Omega_{\mathrm{DM}} h^{2} \simeq 0.1$ and $\Omega_{B} \simeq 0.045$. In blue and green regions, the dark matter is explained by gravitino and baryon asymmetry is supplied by thermal leptogenesis with an appropriate choice of $\Delta$ and $T_{R}$. In the green region, the non-thermally produced gravitino abundance coincides the observed DM abundance. We should discard the parameter regions shaded by (light)gray color. For gray regions denoted as "unstable $S$ " and "unstable $f$," the SUSY breaking minimum is unstable [31]. For a light gray region "fall into SUSY vacuum," the pseudo-moduli fall into SUSY preserving vacuum along the cosmological evolution and never reaches the SUSY breaking vacuum [35]. We define $\Delta_{\max }$ as the maximum dilution factor available under the condition that the oscillation amplitude is small so that $S$ does not fall into SUSY vacuum. In the region $\Delta_{3 / 2}>\Delta_{\max }$ we cannot obtain a required amount of dilution factor $\Delta_{3 / 2}$ while $S$ successfully reaches the SUSY breaking minimum. Gravitinos are overproduced non-thermally in the gray region " $\Omega_{3 / 2}^{\mathrm{NT}} h^{2}>0.3$."

One should check if a light higgsino scenario is compatible with the constraint from the $\mathrm{BBN}$. If the higgsino mass is so small that the life-time becomes as long as $\mathcal{O}(1)$ sec, the decay may alter the abundance of the light elements. We have checked the BBN constraints in the case of $m_{\tilde{h}}=300 \mathrm{GeV}$ and found that such a light higgsino is cosmologically safe if the gravitino is lighter than $\sim 500 \mathrm{MeV}$. A detailed discussion is given in appendix B.

\section{Summary}

We re-investigated the thermal production of the gravitino in general framework of gauge mediation. Calculating the gravitino production cross section using both the goldstino 

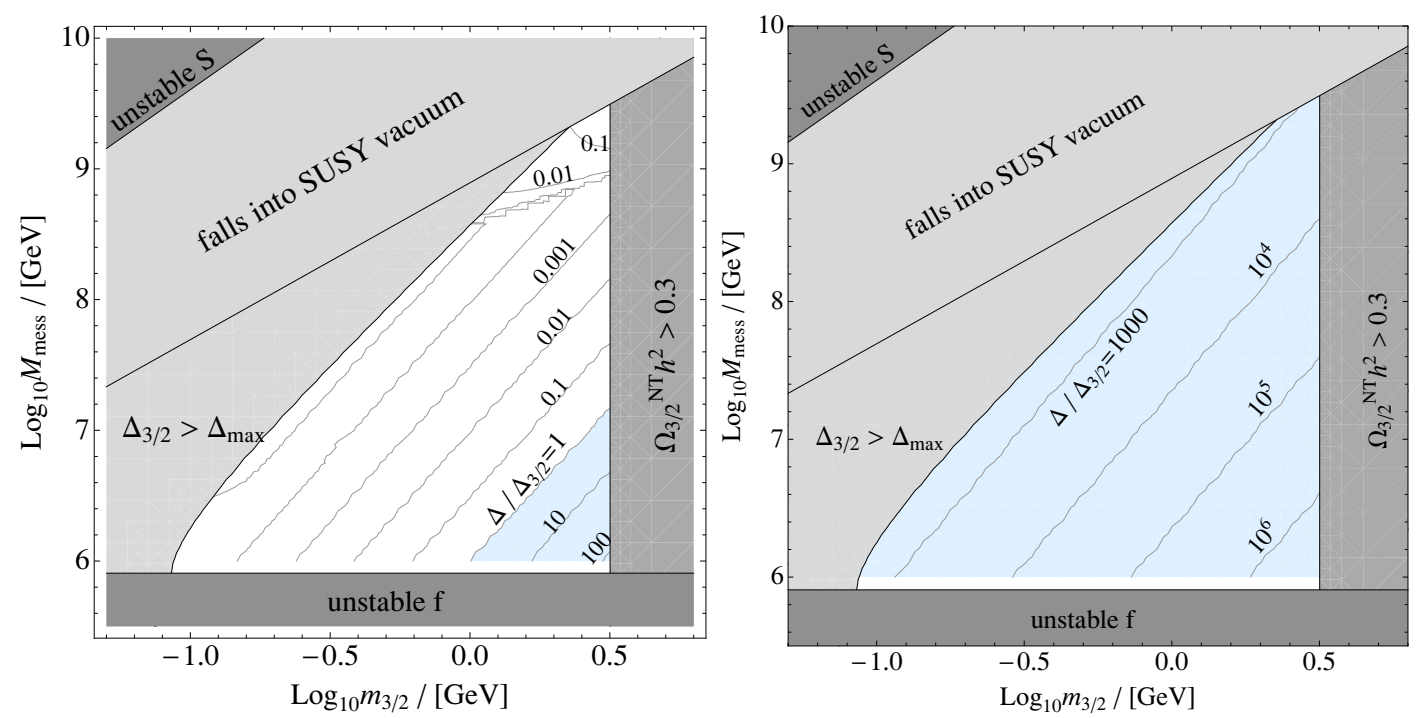

Figure 7. The results of numerical study. In the left(right) figure the initial condition of the position of $S$ right after the inflation is taken to be $S_{0}=\Lambda\left(S_{0}=M_{\mathrm{pl}}\right)$. The required amount of dilution factor and the theoretical prediction are denoted as $\Delta_{3 / 2}$ and $\Delta$. In the blue regions a sizable amount of entropy enough to dilute overabundant gravitino is produced by the decay of $S$. We see that a required amount of dilution factor read off from eq. (3.3) can be always supplied by the decay by choosing an appropriate value of $S_{0}$ from between $\Lambda$ and $M_{\mathrm{pl}}$.

Lagrangian and the supergravity one, we confirmed that the relic abundance become insensitive to the reheating temperature if the temperature of the Universe once exceeds the messenger mass scale. Inspired by this property, we presented a new cosmological scenario; the gravitino dark matter and the thermal leptogenesis are compatible, namely the ratio $\Omega_{3 / 2} / \Omega_{B}$ coincides the observation, $\Omega_{\mathrm{DM}} / \Omega_{B} \sim 5$, with an appropriate value of reheating temperature. To realize the correct absolute value of each quantity, $\Omega_{\mathrm{DM}} h^{2} \simeq 0.1$ and $\Omega_{B} \simeq 0.045$, a late-time entropy release is required, which is automatically supplied by the oscillation energy of the pseudo-moduli.

To make sure that the scenario actually works, we examined cosmological evolution of the pseudo-moduli field in a concrete model of gauge mediation. With an appropriate initial condition, we showed that the oscillation energy of the pseudo-moduli dominates the energy density of the Universe and a sizable amount of entropy needed to fix the energy densities of gravitino and baryon is released by the subsequent decay. The scenario is realized when the gravitino mass is $100 \mathrm{MeV} \lesssim m_{3 / 2} \lesssim 1 \mathrm{GeV}$ and the messenger scale is $10^{6} \mathrm{GeV} \lesssim M_{\text {mess }} \lesssim 10^{9} \mathrm{GeV}$.

Although we have studied the scenario with $M_{\mathrm{SUSY}} \gtrsim 5 \mathrm{TeV}$ to account for the $125 \mathrm{GeV}$ Higgs boson, the higgsino can be as light as $\mathcal{O}(100) \mathrm{GeV}$. Such a light higgsino can be discovered in a future experiments. We have checked that a light higgsino is safe from the $\mathrm{BBN}$ constraints if the gravitino mass is smaller than $\sim 500 \mathrm{MeV}$. 


\section{Acknowledgments}

We would like to thank Fuminobu Takahashi for discussions on gravitino thermal production. RK is supported in part by the Grant-in-Aid for Scientific Research 23740165 of JSPS and 25105011 of MEXT.

\section{A Pseudo-moduli interactions with the MSSM fields}

We summarize the interactions between the pseudo-moduli and the MSSM fields needed to study the decay of $S$. The pseudo-moduli interacts with the MSSM fields through the messenger loop diagrams. The interactions can be read off from the $\langle S\rangle$ dependence of the low energy parameters $[33,34]$. For scalar fields $\tilde{f}$, the effective interaction Lagrangian is written as

$$
\mathcal{L}_{\tilde{f}}=\frac{\left(m_{\mathrm{eff}}^{\tilde{f}}\right)^{2}}{\langle S\rangle} S \tilde{f}^{\dagger} \tilde{f}+\text { h.c. }
$$

The effective mass parameter $\left(m_{\text {eff }}^{\tilde{f}}\right)^{2}$ is a part of the scalar mass that is proportional to $1 /|\langle S\rangle|^{2}$. One element of the scalar mass is the contribution from the gauge mediation,

$$
\left(m_{\mathrm{GM}}^{\tilde{f}}\right)^{2}=\left[\frac{g^{2}}{(4 \pi)^{2}}\right]^{2} \cdot 2 C_{2}\left|\frac{m^{2}}{\langle S\rangle}\right|^{2},
$$

which is induced at the messenger mass scale $M_{\text {mess }}$. If the gauge mediation is the only source of the scalar mass, $m_{\mathrm{eff}}^{\tilde{f}}$ is identical to their mass. In that case, $m_{\mathrm{eff}}^{\tilde{f}}$ is the gauge mediation contribution plus the radiative corrections. In appendix B, we consider a direct coupling between the $S$ field and the Higgs superfields to solve the $\mu$-problem. In that case, $m_{H_{u}}$ consists of two sources; one is from the gauge mediation and the other is from the direct coupling. The latter piece does not depend on $\langle S\rangle$, and has little effect on the effective coupling constant.

As we evaluate the abundance of non-thermally produced higgsino to check the BBN constraint in appendix B, we list the interaction with higgsino,

$$
\mathcal{L}_{\tilde{h}}=-\frac{\mu_{\mathrm{eff}}}{\langle S\rangle} S\left(\overline{h_{d}^{c}} \cdot P_{L} h_{u}\right)+\text { h.c. }
$$

The coefficient $\mu_{\text {eff }}$ is again a part of $\mu$ that is proportional to $1 /\langle S\rangle$. Actually, as we see in appendix B, $\mu$-term is generated at the cutoff scale $\Lambda$ through the Kähler potential eq. (B.1) and it does not have $\langle S\rangle$ dependence. The VEV dependence of $\mu_{\text {eff }}$ appears only through the renormalization group running, and the effect is very small for the $\mu$-term. The effective coupling $\mu_{\mathrm{eff}}$ is suppressed compared to the $\mu$-term, typically

$$
\left|\mu_{\mathrm{eff}}\right| \sim 0.01 \times|\mu| .
$$

Among the effective couplings, the Higgs mass parameter $m_{\mathrm{eff}}^{H_{u}}$ is enhanced by the large renormalization group running [34],

$$
-\left(m_{\mathrm{eff}}^{H_{u}}\right)^{2}=\left(\kappa m_{\tilde{B}}\right)^{2},
$$


with

$$
\kappa \simeq 3-4
$$

Decays of the $S$ field. The $S$ field mainly decays into the MSSM particles. Since the mass parameter $m_{\text {eff }}^{H_{u}}$ is enhanced over other SUSY breaking parameters, the decay rate into the Higgs boson is enhanced. For $m_{S}>2 m_{h}$, the main decay channel turns out to be $S \rightarrow h h, Z Z$ and $W W$, where the gauge bosons are longitudinally polarized [34],

$$
\Gamma_{S \rightarrow h h}+\Gamma_{S \rightarrow Z Z}+\Gamma_{S \rightarrow W W} \simeq \frac{1}{8 \pi m_{S}}\left(\frac{\left(m_{\mathrm{eff}}^{H_{u}}\right)^{2} \sin ^{2} \beta}{\langle S\rangle}\right)^{2} .
$$

Approximating the total decay width $\Gamma_{S}$ by that of main channel, the decay temperature defined in eq. (3.8) is written as

$$
T_{d} \simeq 68 \mathrm{GeV}\left(\frac{g_{*}}{15}\right)^{-1 / 4}\left(\frac{m_{\mathrm{eff}}^{H_{u}}}{5 \mathrm{TeV}}\right)^{2}\left(\frac{m_{\tilde{g}}}{5 \mathrm{TeV}}\right)^{3 / 4}\left(\frac{m_{3 / 2}}{500 \mathrm{MeV}}\right)^{-5 / 4} .
$$

There is also a rare decay mode $S \rightarrow \psi_{3 / 2} \psi_{3 / 2}$, which become important if the gravitino mass is larger than $\sim 1 \mathrm{GeV}$. The decay width is calculated to be [33, 34]

$$
\Gamma_{3 / 2}=\frac{1}{96 \pi} \frac{m_{S}^{3}}{M_{\mathrm{pl}}^{2}}\left(\frac{m_{S}}{m_{3 / 2}}\right)^{2} .
$$

If $S$ dominates the energy density of the Universe, non-thermal gravitino abundance is calculated to be

$$
\Omega_{3 / 2}^{\mathrm{NT}}=\frac{3}{4} m_{3 / 2} \frac{T_{d}}{m_{S}} \times 2 B_{3 / 2} /\left(\rho_{c} / s\right)_{0},
$$

where $\left(\rho_{c} / s\right)_{0} \simeq 1.8 \times 10^{-9} \mathrm{GeV}$ is the critical density divided by the entropy density at present. Approximating the decay temperature as eq. (A.8), the non-thermal gravitino is estimated as

$$
\Omega_{3 / 2}^{\mathrm{NT}} \simeq 0.2\left(\frac{m_{3 / 2}}{2 \mathrm{GeV}}\right)^{9 / 4}\left(\frac{m_{\tilde{g}}}{5 \mathrm{TeV}}\right)^{5 / 4}\left(\frac{m_{\mathrm{eff}}^{H_{u}}}{5 \mathrm{TeV}}\right)^{-2} .
$$

We have used the formula in figure 6 .

\section{B $\mu$-problem and a light higgsino}

Here we present a possible solution to the $\mu$-problem. As we see below, the solution predicts a relatively light higgsino compared to $M_{\text {SUSY }}$. We check whether a light higgsino scenario is allowed by the BBN constraint.

In order to avoid too large $\mu$-term, we assume an approximate Peccei-Quinn (PQ) $\mathrm{U}(1)$ symmetry with a charge assignment $P Q\left(H_{u}\right)=P Q\left(H_{d}\right)=1$. Also, to realize the relation $\mu^{2} \sim m_{H_{u}}^{2}$, we assume the following general interactions between $S$ and the Higgs superfields at the cutoff scale [32],

$$
K^{(\mathrm{Higgs})}=\left(c_{\mu} \frac{S^{\dagger} H_{u} H_{d}}{\Lambda}+\text { h.c. }\right)-c_{H} \frac{S^{\dagger} S\left(H_{u}^{\dagger} H_{u}+H_{d}^{\dagger} H_{d}\right)}{\Lambda^{2}},
$$


where the PQ charge of $S$ is fixed as $P Q(S)=2$. Once the $F$-component of $S$ develops a VEV, $\mu$-term and the Higgs scalar mass terms emerge at the scale $\Lambda$. The relation $\mu^{2} \sim m_{H_{u}}^{2}$, which is needed for satisfying the condition of electroweak symmetry breaking without a serious fine-tuning, naturally realizes if the coefficients $c_{\mu}$ and $c_{H}$ are both $\mathcal{O}(1)$.

Possible origins of the Kähler potential (B.1) are discussed in ref. [32] by studying dynamics of UV models above the cutoff scale $\Lambda$. There, it is found that the coefficients $c_{\mu}$ and $c_{H}$ tend to have a mild hierarchy, and we typically have $\mu / m_{H} \sim 1 / 10$. This hierarchy implies that the Higgs scalar mass parameter $m_{H_{u}}$ tends to be above the order of TeV scale for a moderate value of $\mu$-term, namely $m_{H_{u}} \gtrsim \mathcal{O}(1) \mathrm{TeV}$ for $\mu \gtrsim \mathcal{O}(100) \mathrm{GeV}$.

We do not regard this small hierarchy as catastrophic; actually, this hierarchy is consistent with the relatively heavy Higgs boson mass. In order for the electroweak symmetry to be broken radiatively, the condition

$$
\frac{M_{Z}^{2}}{2} \simeq-\mu^{2}-m_{H_{u}}^{2}(\Lambda)-\delta m_{H_{u}}^{2}
$$

must be satisfied. $\delta m_{H_{u}}^{2}$ is a contribution from the radiative corrections. With positive $m_{H_{u}}^{2}(\Lambda)$ and $\mu^{2} \ll m_{H_{u}}^{2}(\Lambda), \delta m_{H_{u}}^{2}$ must be negative and large to satisfy the condition (B.2), which is realized by the contributions from the stop-loop diagrams if the stop mass $m_{\tilde{t}}$ is large. Large stop mass subsequently induce a large contribution proportional to $m_{\tilde{t}}^{2}$ to the Higgs boson mass again through the stop-loop diagram to realize a relatively heavy Higgs boson. In summary, in this set-up, the $\mu$-problem is ameliorated by the generalized version of the Giudice-Masiero mechanism with the Kähler potential in eq. (B.1), which in turn leads the relatively small $\mu$-term and the relatively heavy Higgs boson mass in accord with $m_{h}=125 \mathrm{GeV}$.

Although it is difficult to discover a SUSY particles at the LHC experiments when $M_{\mathrm{SUSY}} \sim 5 \mathrm{TeV}$, it predicts a light higgsino with $m_{\tilde{h}} \simeq \mathcal{O}(100) \mathrm{GeV}$. Therefore, in this scenario, there is a chance to discover a light higgsino in the future experiment.

The light higgsino in GMSB is subject to the constraints from BBN. The constraints on the primordial abundance of the lightest neutralino $\chi$ is studied in ref. [48]. They analyzed the decay process of the neutralino and presented constraints on $Y_{\chi}=n_{\chi} / s$, the yield of $\chi$, in a Bino-like NLSP case. We use the constraints to derive those for the higgsino.

Since the life-time of a neutralino $\chi$ is approximately proportional to $m_{3 / 2}^{2} / m_{\chi}^{5}$, constraints on the primordial abundance are more severe for larger $m_{3 / 2}$ or smaller $m_{\chi}$. We focus on a case that the mass of NLSP (in our case higgsino) is $300 \mathrm{GeV}$. According to ref. [48], if the gravitino is heavier than $\sim 500 \mathrm{MeV}$, the stringent bound on the bino abundance comes from the overproduction of the Deuterium. For $10 \mathrm{MeV} \lesssim m_{3 / 2} \lesssim 500 \mathrm{MeV}$, the bound is from the overproduction of ${ }^{4} \mathrm{He}$,

$$
\begin{array}{rlrl}
m_{\tilde{B}} Y_{\tilde{B}} & \lesssim 10^{-13} \mathrm{GeV} & (500 \mathrm{MeV} & \left.\lesssim m_{3 / 2} \lesssim 100 \mathrm{GeV}\right), \\
m_{\tilde{B}} Y_{\tilde{B}} \lesssim 10^{-9} \mathrm{GeV} & \left(10 \mathrm{MeV} \lesssim m_{3 / 2} \lesssim 500 \mathrm{MeV}\right) .
\end{array}
$$

The bound is much weaker for $m_{3 / 2} \lesssim 10 \mathrm{MeV}$. We estimate the higgsino abundance in the scenario and check whether a light higgsino is allowed by BBN. 
Higgsinos are produced non-thermally from the decay of the pseudo-moduli,

$$
Y_{\tilde{h}}=\frac{3}{4} \frac{T_{d}}{m_{S}} \times 2 B_{\tilde{h}}
$$

where $B_{\tilde{h}}$ is the branching ratio of the decay process $S \rightarrow \tilde{h} \tilde{h}$ and the decay temperature $T_{d}$ is well approximated by eq. (A.8). $Y_{\tilde{h}}$ depends on two effective couplings: $m_{\text {eff }}^{H_{u}}$ and $\mu_{\text {eff }}$ defined in appendix A. Remaining these parameters, the higgsino abundance is estimated as

$$
m_{\tilde{h}} Y_{\tilde{h}} \simeq 1.2 \times 10^{-7} \mathrm{GeV}\left(\frac{m_{3 / 2}}{500 \mathrm{MeV}}\right)^{-3 / 4}\left(\frac{m_{\mathrm{eff}}^{H_{u}}}{5 \mathrm{TeV}}\right)^{-2}\left(\frac{\mu_{\mathrm{eff}}}{5 \mathrm{GeV}}\right)^{2} .
$$

The abundance of the non-thermally produced higgsinos is decreased by the subsequent annihilation process. This effect can be taken into account by solving the Boltzmann equation,

$$
\dot{n}_{\tilde{h}}+3 H n_{\tilde{h}}=-\langle\sigma v\rangle n_{\tilde{h}}^{2}
$$

where $\langle\sigma v\rangle$ is the thermal averaged annihilation cross section of higgsino [49], ${ }^{3}$

$$
\langle\sigma v\rangle=\frac{g^{4}}{128 \pi \mu^{2}}\left(\frac{3}{2}+\tan ^{2} \theta_{W}+\frac{\tan ^{2} \theta_{W}}{2}\right)
$$

where $\theta_{W}$ is Weinberg angle. The solution of the Boltzmann equation (B.7) is approximated by a simple analytic formula $[34,50]$. In terms of the yield value $Y_{\tilde{h}}=n_{\tilde{h}} / s$,

$$
Y_{\tilde{h}}(T) \simeq\left[\frac{1}{Y_{\tilde{h}}\left(T_{d}\right)}+\sqrt{\frac{8 \pi^{2} g_{*}\left(T_{d}\right)}{45}}\langle\sigma v\rangle M_{\mathrm{pl}}\left(T_{d}-T\right)\right]^{-1} .
$$

If the initial abundance $Y_{\tilde{h}}\left(T_{d}\right)$ produced by the decay of $S$ is large enough, the resultant abundance for $T \ll T_{d}$ is independent of $Y_{\tilde{h}}\left(T_{d}\right)$. In this case, the abundance is estimated by

$$
Y_{\tilde{h}} \simeq 8.2 \times 10^{-13}\left(\frac{15}{g_{*}}\right)^{1 / 2}\left(\frac{10 \mathrm{GeV}}{T_{d}}\right)\left(\frac{10^{-8} \mathrm{GeV}^{-2}}{\langle\sigma v\rangle}\right) .
$$

For higgsino with $m_{\tilde{h}}=300 \mathrm{GeV}$,

$$
m_{\tilde{h}} Y_{\tilde{h}} \simeq 3.9 \times 10^{-11} \mathrm{GeV}\left(\frac{15}{g_{*}}\right)^{3 / 4}\left(\frac{m_{\mathrm{eff}}^{H_{u}}}{5 \mathrm{TeV}}\right)^{-2}\left(\frac{m_{\tilde{g}}}{5 \mathrm{TeV}}\right)^{-3 / 4}\left(\frac{m_{3 / 2}}{500 \mathrm{MeV}}\right)^{5 / 4}\left(\frac{\mu}{300 \mathrm{GeV}}\right)^{2} .
$$

Compared with eq. (B.3) and (B.4), we see that the higgsino abundance is below the BBN constraint for $m_{3 / 2} \lesssim 500 \mathrm{MeV}$ with the help of the annihilation process.

Open Access. This article is distributed under the terms of the Creative Commons Attribution License (CC-BY 4.0), which permits any use, distribution and reproduction in any medium, provided the original author(s) and source are credited.

\footnotetext{
${ }^{3}$ We have not included co-annihilation effects to make a conservative estimate.
} 


\section{References}

[1] M. Dine, W. Fischler and M. Srednicki, Supersymmetric technicolor, Nucl. Phys. B 189 (1981) 575 [inSPIRE].

[2] S. Dimopoulos and S. Raby, Supercolor, Nucl. Phys. B 192 (1981) 353 [inSPIRE].

[3] M. Dine and W. Fischler, A phenomenological model of particle physics based on supersymmetry, Phys. Lett. B 110 (1982) 227 [INSPIRE].

[4] M. Dine and W. Fischler, A supersymmetric GUT, Nucl. Phys. B 204 (1982) 346 [InSPIRE].

[5] C.R. Nappi and B.A. Ovrut, Supersymmetric extension of the $\mathrm{SU}(3) \times \mathrm{SU}(2) \times \mathrm{U}(1)$ model, Phys. Lett. B 113 (1982) 175 [inSPIRE].

[6] L. Álvarez-Gaumé, M. Claudson and M.B. Wise, Low-energy supersymmetry, Nucl. Phys. B 207 (1982) 96 [INSPIRE].

[7] S. Dimopoulos and S. Raby, Geometric hierarchy, Nucl. Phys. B 219 (1983) 479 [InSPIRE].

[8] M. Dine and A.E. Nelson, Dynamical supersymmetry breaking at low-energies, Phys. Rev. D 48 (1993) 1277 [hep-ph/9303230] [INSPIRE].

[9] M. Dine, A.E. Nelson, Y. Nir and Y. Shirman, New tools for low-energy dynamical supersymmetry breaking, Phys. Rev. D 53 (1996) 2658 [hep-ph/9507378] [INSPIRE].

[10] M. Dine, A.E. Nelson and Y. Shirman, Low-energy dynamical supersymmetry breaking simplified, Phys. Rev. D 51 (1995) 1362 [hep-ph/9408384] [INSPIRE].

[11] P. Draper, P. Meade, M. Reece and D. Shih, Implications of a $125 \mathrm{GeV}$ Higgs for the MSSM and low-scale SUSY breaking, Phys. Rev. D 85 (2012) 095007 [arXiv:1112.3068] [INSPIRE].

[12] T. Moroi, H. Murayama and M. Yamaguchi, Cosmological constraints on the light stable gravitino, Phys. Lett. B 303 (1993) 289 [INSPIRE].

[13] M. Kawasaki and T. Moroi, Gravitino production in the inflationary universe and the effects on big bang nucleosynthesis, Prog. Theor. Phys. 93 (1995) 879 [hep-ph/9403364] [InSPIRE].

[14] T. Moroi, Effects of the gravitino on the inflationary universe, hep-ph/9503210 [INSPIRE].

[15] A. de Gouvêa, T. Moroi and H. Murayama, Cosmology of supersymmetric models with low-energy gauge mediation, Phys. Rev. D 56 (1997) 1281 [hep-ph/9701244] [INSPIRE].

[16] M. Bolz, W. Buchmüller and M. Plümacher, Baryon asymmetry and dark matter, Phys. Lett. B 443 (1998) 209 [hep-ph/9809381] [INSPIRE].

[17] M. Bolz, A. Brandenburg and W. Buchmüller, Thermal production of gravitinos, Nucl. Phys. B 606 (2001) 518 [Erratum ibid. B 790 (2008) 336] [hep-ph/0012052] [INSPIRE].

[18] J. Pradler and F.D. Steffen, Thermal gravitino production and collider tests of leptogenesis, Phys. Rev. D 75 (2007) 023509 [hep-ph/0608344] [INSPIRE].

[19] J. Pradler and F.D. Steffen, Constraints on the reheating temperature in gravitino dark matter scenarios, Phys. Lett. B 648 (2007) 224 [hep-ph/0612291] [INSPIRE].

[20] V.S. Rychkov and A. Strumia, Thermal production of gravitinos, Phys. Rev. D 75 (2007) 075011 [hep-ph/0701104] [INSPIRE].

[21] M. Kawasaki, K. Kohri and T. Moroi, Big-bang nucleosynthesis and hadronic decay of long-lived massive particles, Phys. Rev. D 71 (2005) 083502 [astro-ph/0408426] [INSPIRE]. 
[22] M. Fukugita and T. Yanagida, Baryogenesis without grand unification, Phys. Lett. B 174 (1986) 45 [InSPIRE].

[23] S. Davidson and A. Ibarra, A lower bound on the right-handed neutrino mass from leptogenesis, Phys. Lett. B 535 (2002) 25 [hep-ph/0202239] [INSPIRE].

[24] G. Giudice, A. Notari, M. Raidal, A. Riotto and A. Strumia, Towards a complete theory of thermal leptogenesis in the SM and MSSM, Nucl. Phys. B 685 (2004) 89 [hep-ph/0310123] [INSPIRE].

[25] W. Buchmüller, R. Peccei and T. Yanagida, Leptogenesis as the origin of matter, Ann. Rev. Nucl. Part. Sci. 55 (2005) 311 [hep-ph/0502169] [InSPIRE].

[26] W. Buchmüller, P. Di Bari and M. Plümacher, Leptogenesis for pedestrians, Annals Phys. 315 (2005) 305 [hep-ph/0401240] [INSPIRE].

[27] G. 't Hooft and M. Veltman, Scalar one loop integrals, Nucl. Phys. B 153 (1979) 365 [INSPIRE].

[28] K. Choi, K. Hwang, H.B. Kim and T. Lee, Cosmological gravitino production in gauge mediated supersymmetry breaking models, Phys. Lett. B 467 (1999) 211 [hep-ph/9902291] [INSPIRE].

[29] K. Jedamzik, M. Lemoine and G. Moultaka, Gravitino dark matter in gauge mediated supersymmetry breaking, Phys. Rev. D 73 (2006) 043514 [hep-ph/0506129] [INSPIRE].

[30] I. Dalianis, Gravitino dark matter production at finite temperature, JHEP 11 (2013) 162 [arXiv: 1304.7673] [INSPIRE].

[31] R. Kitano, Gravitational gauge mediation, Phys. Lett. B 641 (2006) 203 [hep-ph/0607090] [INSPIRE].

[32] M. Ibe and R. Kitano, Sweet spot supersymmetry, JHEP 08 (2007) 016 [arXiv:0705.3686] [INSPIRE].

[33] M. Ibe and R. Kitano, Gauge mediation in supergravity and gravitino dark matter, Phys. Rev. D 75 (2007) 055003 [hep-ph/0611111] [INSPIRE].

[34] K. Hamaguchi, R. Kitano and F. Takahashi, Non-thermal gravitino dark matter in gauge mediation, JHEP 09 (2009) 127 [arXiv:0908.0115] [INSPIRE].

[35] H. Fukushima, R. Kitano and F. Takahashi, Cosmologically viable gauge mediation, JHEP 02 (2013) 140 [arXiv:1209.1531] [INSPIRE].

[36] I. Dalianis and Z. Lalak, Cosmological vacuum selection and metastable SUSY breaking, JHEP 12 (2010) 045 [arXiv: 1001.4106] [INSPIRE].

[37] I. Dalianis and Z. Lalak, Thermally favourable gauge mediation, Phys. Lett. B 697 (2011) 385 [arXiv: 1012.3157] [InSPIRE].

[38] S.A. Abel, C.-S. Chu, J. Jaeckel and V.V. Khoze, SUSY breaking by a metastable ground state: why the early universe preferred the non-supersymmetric vacuum, JHEP 01 (2007) 089 [hep-th/0610334] [INSPIRE].

[39] N.J. Craig, P.J. Fox and J.G. Wacker, Reheating metastable O'Raifeartaigh models, Phys. Rev. D 75 (2007) 085006 [hep-th/0611006] [INSPIRE].

[40] W. Fischler, V. Kaplunovsky, C. Krishnan, L. Mannelli and M.A. Torres, Meta-stable supersymmetry breaking in a cooling universe, JHEP 03 (2007) 107 [hep-th/0611018] [INSPIRE]. 
[41] S.A. Abel, J. Jaeckel and V.V. Khoze, Why the early universe preferred the non-supersymmetric vacuum: part II, JHEP 01 (2007) 015 [hep-th/0611130] [INSPIRE].

[42] L. Anguelova, R. Ricci and S. Thomas, Metastable SUSY breaking and supergravity at finite temperature, Phys. Rev. D 77 (2008) 025036 [hep-th/0702168] [INSPIRE].

[43] C. Papineau, Finite temperature behaviour of the ISS-uplifted KKLT model, JHEP 05 (2008) 068 [arXiv:0802.1861] [INSPIRE].

[44] R. Auzzi, S. Elitzur and A. Giveon, On uplifted SUSY-breaking vacua and direct mediation in generalized SQCD, JHEP 03 (2010) 094 [arXiv: 1001.1234] [INSPIRE].

[45] A. Katz, On the thermal history of calculable gauge mediation, JHEP 10 (2009) 054 [arXiv:0907.3930] [INSPIRE].

[46] E. Moreno and F. Schaposnik, R-symmetry and supersymmetry breaking at finite temperature, JHEP 10 (2009) 007 [arXiv:0908.2770] [INSPIRE].

[47] A. Ferrantelli and J. McDonald, Cosmological evolution of scalar fields and gravitino dark matter in gauge mediation at low reheating temperatures, JCAP 02 (2010) 003 [arXiv:0909.5108] [INSPIRE].

[48] M. Kawasaki, K. Kohri, T. Moroi and A. Yotsuyanagi, Big-bang nucleosynthesis and gravitino, Phys. Rev. D 78 (2008) 065011 [arXiv:0804.3745] [INSPIRE].

[49] N. Arkani-Hamed, A. Delgado and G. Giudice, The well-tempered neutralino, Nucl. Phys. B 741 (2006) 108 [hep-ph/0601041] [INSPIRE].

[50] M. Fujii and K. Hamaguchi, Nonthermal dark matter via Affleck-Dine baryogenesis and its detection possibility, Phys. Rev. D 66 (2002) 083501 [hep-ph/0205044] [INSPIRE].

[51] J. Heisig, Gravitino LSP and leptogenesis after the first LHC results, arXiv:1310.6352 [INSPIRE]. 